\title{
Diverse mechanisms of IRF5 action in inflammatory responses
}

\author{
Tariq E Khoyratty and Irina A Udalova
}

\begin{abstract}
Interferon regulatory factor 5 (IRF5) is a key signal-dependent transcription factor in myeloid cells. Its expression is induced by granulocyte-macrophage colony stimulating factor and interferon-gamma. IRF5 protein is further activated in response to stimulation, translocating to the nucleus where it mediates inflammatory responses. IRF5 is capable of both the up-regulation of pro-inflammatory genes and repressing anti-inflammatory mediators, thus polarising macrophages to a proinflammatory phenotype. We discuss IRF5 interactions with a wide range of transcriptional regulators that give rise to its diverse effects at the level of chromatin.
\end{abstract}

\section{Introduction}

\section{Interferon Regulatory Factors (IRFs)}

The interferon regulatory factor (IRF) transcription factor family comprises nine members in mammals (IRF1-9), which were originally described for their role in the induction of type I interferons (IFNs) (Miyamoto et al., 1988) (Wathelet et al., 1998). IRFs bind to consensus interferon stimulated response element (ISRE) motifs, consisting of two GAAA repeats, via their shared N-terminal DNA binding domain (DBD) (Tanaka et al., 1993). ISRE motifs are widely enriched at regulatory sites of immune genes, leading to the functional diversity of the IRFs and their key roles as master regulators of the innate immune responses (Honda and Taniguchi, 2006). The role of IRFs in the context of immune cell activation has been extensively studied and of the IRFs four have been implicated as core molecules in the induction of type I IFNs: IRF1, IRF3, IRF5, and IRF7. IRFs are activated in response to a diverse array of pattern recognition receptors (PRRs), including Toll like receptors (TLR) and cytosolic PRRs, in response to pathogen associated molecular patterns (PAMPs). In the case of TLR4 ligation by lipopolysaccharide (LPS) two major signaling pathways are activated, mediated by the key adaptor molecules MyD88 and TRIF. Activation of IRF1 and IRF5 is dependent on their binding MyD88, whereas IRF3 and IRF7 are activated downstream of TRIF via TBK1. Phosphorylation of these IRFs initiates their homo- and hetero-dimerization and translocation to the nucleus, where they elicit expression of type I IFNs (Honda and Taniguchi, 2006). IRF1 and IRF5 have both been demonstrated to contribute to type I IFN gene expression (Barnes et al., 2001) (Yie et al., 1999) (Fujita et al., 1989). However, Newcastle disease virus infection of IRF1-deficient fibroblasts, vesicular stomatitis virus infection of fibroblasts from IRF5 $5^{-1-}$ knockout mice and $\mathrm{CpG}$ stimulation of IRF5 ${ }^{-1-}$ plasmacytoid dendritic cells ( $p D C s$ ) indicate that these factors aren't crucial for type I IFN induction as IFN $\beta$ \& IFN $\alpha$ are still up-regulated in their absence (Matsuyama et al., 1993) (Yanai et al., 2007) (Takaoka et al., 2005). Thus implicating the highly homologous IRF3 and IRF7 as the core IRFs in mediating anti-viral responses. IRF5 on the other hand has much broader activity. It is widely expressed by immune cells, including macrophages, B cells, monocytes, and dendritic cells (Heng et al., 2008), and can be used as a marker of inflammatory macrophages both in vitro and in vivo (Weiss et al., 2013).

Studies in $/ r f 5^{-/}$mice highlight the importance of IRF5 to macrophage function. Irf5 $5^{- \text {mice }}$ are resistant to lethal endotoxic shock (Takaoka et al., 2005). This is due to a crucial role for IRF5 in promoting pro-inflammatory responses as a consequence of TLR activation. Stimulation of splenic macrophages with ligands for TLR3, TLR4, TLR7/8, and TLR9 all result in in IRF5 activation, via its direct interaction with the MyD88 and TRAF6 adaptors, which are required for IRF5 phosphorylation and ubiquitination (Takaoka et al., 2005). Interestingly, IRF4 binds to the same site on MyD88 as IRF5, competing with IRF5 for activation (Negishi et al., 2005). In addition, Lyn, a Src family kinase, was shown to physically interacted with IRF5 and inhibit its activation via the TLR-MyD88 pathway (Ban et al., 2016). IRF5 contains two nuclear localization signals (NLS), IRF5 phosphorylation results in the $5^{\prime}$ NLS becoming exposed, mediating the nuclear translocation and retention of IRF5 in activated cells (Barnes et al., 2002). The precise mechanisms of IRF5 activation are reviewed fully by Ryzhakov et al. (Ryzhakov et al., 2015). Once activated IRF5 reaches the nucleus where it mediates transcription of 
inflammatory cytokines such as TNF, IL12b, and IL-6, all of which are drastically reduced in cells derived from $/ \mathrm{rf5}^{-/-}$mice (Takaoka et al., 2005). The transcriptional activity of IRF4 closely mirrors that of IRF5, downregulating IRF5 target genes (Negishi et al., 2005), the IRF4-IRF5 axis is therefore integral for the polarization of macrophage responses.

\section{$\underline{\text { IRF5 and Macrophages in Inflammation }}$}

Macrophage inflammatory responses have typically been defined as fitting either the M1 (classically) or M2 (alternatively) activated phenotypes. However, appreciation of the in vivo complexity of the milieu of polarizing factors macrophages are exposed to and the resulting heterogeneity in macrophage responses is growing (Murray et al., 2014). The classic view of macrophage activation corresponds to in vitro polarization with pro-inflammatory stimuli such as IFN $\gamma$ and LPS, leading to robust inflammatory responses, which are characterized by key cytokines such as TNF and IL12b; or alternative treatment with IL-4 or IL-13, which stimulates IL-10 secretion. These alternatively activated macrophages are more capable at handling parasitic infection, wound healing, and repair related functions than their inflammatory counterparts, which mediate sterilizing inflammation for the efficient killing of bacterial and intracellular pathogens. Within this context IRF5 has been shown to be a crucial mediator of inflammatory macrophages in vivo and classical macrophage activation in vitro.

IRF5 expression is induced during differentiation from human monocytes and murine bone marrow to macrophages (Krausgruber et al., 2011). Its activation leads to TNF, IL-6, IL-12 and IL-23 secretion whilst simultaneously repressing IL-10 and TGF-? (Krausgruber et al., 2011) (Ouyang et al., 2007) (Dalmas et al., 2015) (Figure 1). IRF5 expression is therefore integral for the inflammatory polarization of macrophages and has an immunomodulatory role, promoting Th1 and Th17 responses. Conversely, IRF4 has been implicated in alternative macrophage activation, its induction leading to the expression of key M2 markers (Satoh et al., 2010) and dampening of inflammatory responses. Supporting this IRF5 has been identified as one of the top expression quantitative trait loci's associated with TLR4 induced human monocyte inflammatory responses (Kim et al., 2014). Further single nucleotide polymorphisms (SNPs) affecting Irf5 expression have been linked with increased susceptibility to: systemic lupus erythematosus (SLE), rheumatoid arthritis, and inflammatory bowel disease (Eames et al., 2016). This has been reinforced by work demonstrating the pro-inflammatory activity of IRF5 in vivo: in acute antigen-induced arthritis (AIA) $/ r f 5^{-1-}$ mice have reduced neutrophil influx into the joint protecting it from damage, and in the pristine model of SLE the T cell response in $/ \mathrm{rf}^{\%}$ mice is shifted from a Th1 to Th2 phenotype, this was coupled with decreased pDC recruitment and IFNa levels. Interestingly, both of these studies demonstrate an effect of IRF5 on recruitment of effector cells implicating a role for IRF5 in the secretion of chemokines. Homeostatic macrophage functions are also impacted by IRF5, in the lung global IRF5 depletion results in increased extracellular matrix deposition and fibrosis, and in atherosclerosis $\operatorname{Irf5} 5^{-/-}$mice exhibited decreased lesion and necrotic cores sizes, due to the increased efferocytosis and clearance of debris by macrophages derived from the $\operatorname{Irf5} 5^{-1-}$ compared to wild type macrophages (Seneviratne et al., 2017). Together these data can be taken to indicate that IRF5 promotes a pro-inflammatory macrophage phenotype at the expense of their homeostatic activity.

Figure 1. IRF5 is a key mediator of inflammatory macrophages. IRF5 induction leads to TNF, IL-6, IL12 and IL-23 secretion whilst simultaneously repressing IL-10, TGF-b. In vitro macrophages can be polarised to a classic (M1) state or alternatively activated (M2) phenotype by culture with either LPS and IFN-g or IL-4 and IL-13. IFN-? and GM-CSF induce expression of IRF5 mRNA, while LPS induces post-translational modifications of IRF5 protein. 


\section{IRF5 Function at Promoters}

The capacity of macrophages to respond dynamically to change is dependent not only on the inducing stimuli and the reactive transcription factors, but also on the chromatin state of the responding cell. It was first noted that NFKB could bind to some genes very rapidly, but was delayed in reaching others, indicating that chromatin remodeling was required to reveal the cognate binding site (Saccani et al., 2001). Reflecting this, macrophage gene transcription can be broadly divided into two categories; primary response genes (PRGs), the promoters of which are associated with permissive chromatin and are rapidly induced in response to stimuli, and secondary response genes (SRGs), requiring chromatin remodeling at promoters for their induction. DNA has an inherent affinity for the core histone subunits $(\mathrm{H} 1, \mathrm{H} 2 \mathrm{a}, \mathrm{H} 2 \mathrm{~b}, \mathrm{H} 3$, and $\mathrm{H} 4)$ allowing the spontaneous assembly and packaging of DNA around nucleosomes in a highly ordered structure, with 147b.p. DNA wrapping around each nucleosome, sequestering it from damage and preventing the binding of other factors. Cis-regulatory regions have an increased affinity for nucleosomes, whereas promoters of housekeeping and constitutively active genes are often associated with very high CG dinucleotide enrichment, these features are known as CpG islands and constrain the ability of DNA to bind nucleosomes, resulting in nucleosome depletion (Fenouil et al., 2012) (Ramirez-Carrozzi et al., 2009). Approximately seventy percent of mammalian gene promoters contain $\mathrm{CpG}$ islands, allowing dynamic turnover of histones and competition with transcription factors for binding.

In macrophages, many PRGs have accessible promoters and are basally associated with Pol II and markers of active gene transcription such as histone3 lysine27 (H3K27) acetylation (Ramirez-Carrozzi et al., 2009) (Adelman et al., 2009) (Hargreaves et al., 2009) (Bhatt et al., 2012). Genes falling in this category are poised for transcription and are rapidly induced after sufficient stimulus. Whereas promoters at SRGs are dependent on nucleosome remodeling and protein synthesis for increased chromatin accessibility and transcription (Ramirez-Carrozzi et al., 2006) (Ramirez-Carrozzi et al., 2009). However, it is worth noting that the rate of gene induction by LPS doesn't closely correlate with promoter CpG content (Bhatt et al., 2012), with both PRGs and SRGs having a high number of promoters with CpG islands. This indicates that more is at play in terms of chromatin remodeling and gene induction than solely promoter architecture.

IRF5 binds to promoters of both rapidly induced PRGs (such as TNF and IL1a) and to SRGs (e.g. IL6, IL12b) as a consequence of LPS stimulation, leading to large increases in Pol II recruitment (Krausgruber et al., 2011) (Saliba et al., 2014). Of the IRF5 up-regulated genes, those exhibiting the highest increases in expression with LPS stimulation are also targets of NF-? R RelA, the binding sites of which coincide with those of IRF5 and are centered on the promoters of these genes (Figure 2A). Interestingly, RelA peaks not overlapping with IRF5 are more evenly distributed across both LPS upand down- regulated genes (Saliba et al., 2014). This fits with other reports indicating that the majority of RelA binding sites are not associated with transcriptional changes (Lim et al., 2007). Similar IRF5-RelA interactions were observed at the TNF loci in monocyte-derived dendritic cells (mDDCs) (Krausgruber et al., 2010). Removal of RelA from this system results in a reduction in IRF5 binding and subsequent Pol II recruitment at $I L-6, I L-1 a$, and TNF promoters (Saliba et al., 2014), it is therefore likely that ReIA aids in IRF5 recruitment to these regions. This poses an additional mechanism of regulation, with IRF5 activation and binding to promoters being required for high levels of Pol II recruitment and gene expression.

Figure 2. Mechanisms of IRF5 action. A) IRF5 acts co-operatively with RelA to recruit Pol II to the promoters of pro-inflammatory genes. B) IRF5 interacts with epigenetic co-activators such as $\mathrm{CBP} / \mathrm{p} 300$, the recruitment of which results in $\mathrm{H} 3 \mathrm{~K} 27$ acetylation and is associated with the activation of enhancers. C) IRF5 may participate in long distance interactions between promoters and enhancers of target genes. D) IRF5 also interacts with negative epigenetic modulators such as SETDB1, which acts to deposit repressive histone marks mediating decreased gene expression. 
Distal cis-regulatory elements known as enhancers offer a dynamic platform for transcription factor binding, which is subject to much regulation and change; leading to the wide heterogeneity exhibited by macrophages. Closed chromatin regions are characterized by DNA methylation and the repressive H3K27me3 mark. These areas of heterochromatin are not accessible to low-order signal-dependent transcription factors (SD-TFs), which require exposed DNA to bind (Lone et al., 2013). However, the pioneer factor PU.1 is able to bind partially exposed DNA in heterochromatin, in a sequence-specific manner, facilitating nucleosome removal and maintaining nucleosome depletion at these sites (Barozzi et al., 2014) (Soufi et al., 2015). These distal regulatory elements exhibiting diminished nucleosome occupancy and PU.1 binding are classed as enhancers, acting as platforms for subsequent binding of inducible transcription factors (Schmidt et al., 2016) (Ghisletti et al., 2010). PU.1 is an Ets transcription factor family member and is well known as a lineage-determining transcription factor, induced early in hematopoiesis due to its requirement for both myeloid and lymphoid cell development (Olson et al., 1995) (Back et al., 2005) (Nutt et al., 2005). However, PU.1 expression is ten-fold higher in macrophages than B cells (DeKoter and Singh, 2000) and is crucial for the delineation of macrophage-specific enhancers with the H3K4me1 histone mark (Heinz et al., 2010) (Ghisletti et al., 2010), which distinguishes them from promoters (Heintzman et al., 2007). PU.1 designated enhancers encompass the core macrophage regulatory landscape, which is tailored in accordance with micro environmental signals to yield wide heterogeneity and specialization dependent on tissue location, prior exposure to inflammatory signals, and corresponding transcription factor expression profile (Lavin et al., 2014) (Ostuni et al., 2013). In the context of inflammatory responses PU.1 has been shown to be crucial in the recruitment of chromatin remodelers BAF and PBAF (of the SWI/SNF family), to establish and maintain low nucleosome occupancy of enhancers corresponding to pro-inflammatory cytokines (McAndrew et al., 2016). PU.1 therefore designates and maintains the landscape in which SD-TFs such as IRF5 are free to operate, supporting the specific initiation of gene expression upon stimulation and the successful recruitment of transcription factors. Of relevance, the regions of simultaneous IRF5 and RelA binding described above are located in the PU.1-marked regulatory elements (promoters and enhancers) of inflammatory genes, 70\% of which are induced upon LPS stimulation (Saliba et al., 2014). Moreover, the IRF5:RelA cistrome is best explained by the presence of consensus NF-kB and noncanonical composite PU.1:ISRE sites (Saliba et al., 2014).

In response to activation $\mathrm{H} 3 \mathrm{~K} 4 \mathrm{me} 1$ defined enhancers and latent, unmarked enhancers (Ostuni et al., 2013), undergo modification mediated by inducible recruitment of the histone acetlytransferases (HATs) p300/CBP (Ghisletti et al., 2010), and GCN5/PCAF (Schiltz et al., 1999)1. Resulting in acetylation of histones H3K27 (H3K27AC) and H3K9 (H3K9Ac), by either p300/CBP or PCAF/GCN5, respectively (Jin et al., 2011). Little is known about the precise function of these markers, but distinct roles have been reported, with H3K27ac at specific enhancers being required for target gene activation (Jin et al., 2011). H3K27ac has further been shown to be associated with active enhancers in macrophages (Kaikkonen et al., 2013), and is commonly used as a marker for enhancer activation (Glass and Natoli, 2015) (Zhu et al., 2013). Intriguingly CBP and p300 interact directly with IRF5, as does the histone deacetylase HDAC1 (Feng et al., 2010), suggesting that IRF5 may contribute to the recruitment of epigenetic modulators to enhancers, eliciting histone acetylation, activation, and subsequent transcription of corresponding genes (Figure 2B).

The contribution of distal enhancers to gene induction is believed to be mediated via a process known as looping, in which the intervening DNA is looped out, bringing the enhancer and associated promoter into close proximity and aiding the assembly of the transcriptional pre-initiation complex (García-González et al., 2016). The specificity of an enhancer to its cognate promoter is mediated, in part, by the transcription factors and epigenetic mediators binding to the cis-regulatory regions, which participate in protein-protein interactions (van Arensbergen et al., 2014). IRF5 has been shown to be required for sustained high levels of TNF expression in human mDDCs, and it is hypothesized that the observed binding of IRF5 to both the promoter and to an enhancer (downstream of the TNF gene) mediates looping and augmented gene expression (Krausgruber et al., 2010) (Figure 2C).

Aside from its pro-inflammatory capacity, IRF5 is also able to as a negative regulator of transcription, particularly in instances in which IRF5 binding is observed in the absence of RelA (Saliba et al., 2014). This may be through the ability of IRF5 to recruit transcriptional repressors such as HDAC1 (Feng et 
al., 2010) and TRIM28 (Eames et al., 2012). TRIM28 is associated with the establishment of heterochromatin and resultant gene silencing via interactions with HP1 (Sripathy et al., 2006). TRIM28 is also associated with transient transcriptional changes, modifying histone tail residues, mediated by recruitment of SETDB1 (Schultz et al., 2002). SETDB1 is a histone methyltransferase, responsible for the establishment of the repressive H3K9me3 mark. In the context of TNF it has been shown that IRF5 mediates fine-tuned regulation of this cytokine by a combination of binding cis-regulatory elements and the associated Pol II recruitment (Krausgruber et al., 2010), and epigenetic modulation of the underlying histones by SETDB1 recruitment and H3K9 methylation to limit aberrant over-production of TNF (Eames et al., 2012) (Figure 2D).

\section{Conclusions \& Perspectives}

IRF5 is a potent pro-inflammatory transcription factor, orchestrating transcription of inflammatory mediators and polarizing macrophages to a Th1/17 inducing phenotype. This translates to animal models of disease, in which IRF5 function has been studied utilizing IRF5 ${ }^{-1-}$ mice. IRF5 has been show to play a key role in both antigen-induced arthritis (AIA) and LPS-driven lung injury, in which mice lacking IRF5 have decreased neutrophil influx in the acute phase (Weiss et al., 2015). Similar reduction in neutrophil recruitment was observed in the $\mathrm{K} / \mathrm{B} \times \mathrm{N}$ serum-transfer-induced arthritis model, mice lacking IRF5 in this model also have reduced severity of arthritis (Duffau et al., 2015). Additionally, $\mathrm{IRF5}^{-/-}$mice exhibit reduced Th1, Th17 and $\gamma \delta$ T cells in the chronic phase of AIA (Weiss et al., 2015). IRF5 expression in macrophages is pathogenic in diet-induced obesity (Dalmas et al., 2015), but protective against allergic airway inflammation (Byrne et al., 2016).

In humans IRF5 single nucleotide polymorphisms are associated with a range of autoimmune diseases, including: systemic lupus erythematosus, rheumatoid arthritis, and inflammatory bowel disease (Eames et al., 2016). Additionally, IRF5 has been reported as one of the most significant expression quantitative trait loci (eQTL) in human myeloid cells stimulated with LPS (Lee et al., 2014) (Kim et al., 2014) contributing to differences in immune responses; highlighting its role as a key regulator of TLR induced inflammatory responses. IRF5 therefore presents a potential target for disease modifying drugs for the treatment of autoimmune diseases, offering increased specificity and selectivity over broad-spectrum targets.

\section{Acknowledgements}

This work was supported by the Kennedy Institute Trustees' Research Fund (T.E.K. and I.A.U.) and Arthritis Research UK (I.A.U).

\section{Figure legends}

Figure 1. IRF5 is a key mediator of inflammatory macrophages. IRF5 induction leads to TNF, IL-6, IL12 and IL-23 secretion whilst simultaneously repressing IL-10, TGF-b. In vitro macrophages can be polarised to a classic (M1) state or alternatively activated (M2) phenotype by culture with either LPS and IFN-g or IL-4 and IL-13. IFN-g and GM-CSF induce expression of IRF5 mRNA, while LPS induces post-translational modifications of IRF5 protein.

Figure 2. Mechanisms of IRF5 action. A) IRF5 acts co-operatively with RelA to recruit Pol II to the promoters of pro-inflammatory genes. B) IRF5 interacts with epigenetic co-activators such as $\mathrm{CBP} / \mathrm{p} 300$, the recruitment of which results in $\mathrm{H} 3 \mathrm{~K} 27$ acetylation and is associated with the activation of enhancers. C) IRF5 may participate in long distance interactions between promoters and enhancers of target genes. D) IRF5 also interacts with negative epigenetic modulators such as SETDB1, which acts to deposit repressive histone marks mediating decreased gene expression.

\section{$\underline{\text { References }}$}

Adelman, K., Kennedy, M.A., Nechaev, S., Gilchrist, D.A., Muse, G.W., Chinenov, Y., Rogatsky, I., 2009. 
Immediate mediators of the inflammatory response are poised for gene activation through RNA polymerase II stalling. Proc Natl Acad Sci USA 106, 18207-18212. doi:10.1073/pnas.0910177106

Back, J., Allman, D., Chan, S., Kastner, P., 2005. Visualizing PU.1 activity during hematopoiesis. Exp. Hematol. 33, 395-402. doi:10.1016/j.exphem.2004.12.010

Ban, T., Sato, G.R., Nishiyama, A., Akiyama, A., Takasuna, M., Umehara, M., Suzuki, S., Ichino, M., Matsunaga, S., Kimura, A., Kimura, Y., Yanai, H., Miyashita, S., Kuromitsu, J., Tsukahara, K., Yoshimatsu, K., Endo, I., Yamamoto, T., Hirano, H., Ryo, A., Taniguchi, T., Tamura, T., 2016. Lyn Kinase Suppresses the Transcriptional Activity of IRF5 in the TLR-MyD88 Pathway to Restrain the Development of Autoimmunity. Immunity 45, 319-332. doi:10.1016/j.immuni.2016.07.015

Barnes, B.J., Kellum, M.J., Field, A.E., Pitha, P.M., 2002. Multiple regulatory domains of IRF-5 control activation, cellular localization, and induction of chemokines that mediate recruitment of $T$ lymphocytes. Mol. Cell. Biol. 22, 5721-5740. doi:10.1128/MCB.22.16.5721-5740.2002

Barnes, B.J., Moore, P.A., Pitha, P.M., 2001. Virus-specific activation of a novel interferon regulatory factor, IRF-5, results in the induction of distinct interferon alpha genes. J. Biol. Chem. 276, 23382-23390. doi:10.1074/jbc.M101216200

Barozzi, I., Simonatto, M., Bonifacio, S., Yang, L., Rohs, R., Ghisletti, S., Natoli, G., 2014. Coregulation of Transcription Factor Binding and Nucleosome Occupancy through DNA Features of Mammalian Enhancers. Molecular Cell 54, 844-857. doi:10.1016/j.molcel.2014.04.006

Bhatt, D.M., Pandya-Jones, A., Tong, A.-J., Barozzi, I., Lissner, M.M., Natoli, G., Black, D.L., Smale, S.T., 2012. Transcript dynamics of proinflammatory genes revealed by sequence analysis of subcellular RNA fractions. Cell 150, 279-290. doi:10.1016/j.cell.2012.05.043

Byrne, A.J., Weiss, M., Mathie, S.A., Walker, S.A., Eames, H.L., Saliba, D., Lloyd, C.M., Udalova, I.A., 2016. A critical role for IRF5 in regulating allergic airway inflammation. Mucosal Immunol 10, 716-726. doi:10.1038/mi.2016.92

Dalmas, E., Toubal, A., Alzaid, F., Blazek, K., Eames, H.L., Lebozec, K., Pini, M., Hainault, I., Montastier, E., Denis, R.G.P., Ancel, P., Lacombe, A., Ling, Y., Allatif, O., Cruciani-Guglielmacci, C., André, S., Viguerie, N., Poitou, C., Stich, V., Torcivia, A., Foufelle, F., Luquet, S., Aron-Wisnewsky, J., Langin, D., Clément, K., Udalova, I.A., Venteclef, N., 2015. Irf5 deficiency in macrophages promotes beneficial adipose tissue expansion and insulin sensitivity during obesity. Nature Medicine 21, 610-618. doi:10.1038/nm.3829

DeKoter, R.P., Singh, H., 2000. Regulation of B lymphocyte and macrophage development by graded expression of PU.1. Science 288, 1439-1441. doi:10.1126/science.288.5470.1439

Duffau, P., Menn-Josephy, H., Cuda, C.M., Dominguez, S., Aprahamian, T.R., Watkins, A.A., Yasuda, K., Monach, P., Lafyatis, R., Rice, L.M., Kenneth Haines, G., Gravallese, E.M., Baum, R., Richez, C., Perlman, H., Bonegio, R.G., Rifkin, I.R., 2015. Promotion of Inflammatory Arthritis by Interferon Regulatory Factor 5 in a Mouse Model. Arthritis Rheumatol 67, 3146-3157. doi:10.1002/art.39321

Eames, H.L., Corbin, A.L., Udalova, I.A., 2016. Interferon regulatory factor 5 in human autoimmunity and murine models of autoimmune disease. Transl Res 167, 167-182. doi:10.1016/j.trsl.2015.06.018

Eames, H.L., Saliba, D.G., Krausgruber, T., Lanfrancotti, A., Ryzhakov, G., Udalova, I.A., 2012. KAP1/TRIM28: an inhibitor of IRF5 function in inflammatory macrophages. Immunobiology 217, 1315-1324. doi:10.1016/j.imbio.2012.07.026

Feng, D., Sangster-Guity, N., Stone, R., Korczeniewska, J., Mancl, M.E., Fitzgerald-Bocarsly, P., Barnes, B.J., 2010. Differential requirement of histone acetylase and deacetylase activities for IRF5mediated proinflammatory cytokine expression. The Journal of Immunology 185, 6003-6012. doi:10.4049/jimmunol.1000482

Fenouil, R., Cauchy, P., Koch, F., Descostes, N., Cabeza, J.Z., Innocenti, C., Ferrier, P., Spicuglia, S., Gut, M., Gut, I., Andrau, J.-C., 2012. CpG islands and GC content dictate nucleosome depletion in a transcription-independent manner at mammalian promoters. Genome Res. 22, 2399-2408. doi:10.1101/gr.138776.112

Fujita, T., Kimura, Y., Miyamoto, M., Barsoumian, E.L., Taniguchi, T., 1989. Induction of endogenous IFN-alpha and IFN-beta genes by a regulatory transcription factor, IRF-1. Nature 337, 270-272. doi:10.1038/337270a0

García-González, E., Escamilla-Del-Arenal, M., Arzate-Mejía, R., Recillas-Targa, F., 2016. Chromatin remodeling effects on enhancer activity. Cell. Mol. Life Sci. 73, 2897-2910. doi:10.1007/s00018016-2184-3 
Ghisletti, S., Barozzi, I., Mietton, F., Polletti, S., De Santa, F., Venturini, E., Gregory, L., Lonie, L., Chew, A., Wei, C.-L., Ragoussis, J., Natoli, G., 2010. Identification and Characterization of Enhancers Controlling the Inflammatory Gene Expression Program in Macrophages. Immunity 32, 1-12. doi:10.1016/j.immuni.2010.02.008

Glass, C.K., Natoli, G., 2015. Molecular control of activation and priming in macrophages. Nat. Immunol. 17, 26-33. doi:10.1038/ni.3306

Hargreaves, D.C., Horng, T., Medzhitov, R., 2009. Control of Inducible Gene Expression by SignalDependent Transcriptional Elongation. Cell 138, 129-145. doi:10.1016/j.cell.2009.05.047

Heintzman, N.D., Stuart, R.K., Hon, G., Fu, Y., Ching, C.W., Hawkins, R.D., Barrera, L.O., Van Calcar, S., Qu, C., Ching, K.A., Wang, W., Weng, Z., Green, R.D., Crawford, G.E., Ren, B., 2007. Distinct and predictive chromatin signatures of transcriptional promoters and enhancers in the human genome. Nature Genetics 39, 311-318. doi:10.1038/ng1966

Heinz, S., Benner, C., Spann, N., Bertolino, E., Lin, Y.C., Laslo, P., Cheng, J.X., Murre, C., Singh, H., Glass, C.K., 2010. Simple Combinations of Lineage-Determining Transcription Factors Prime cisRegulatory Elements Required for Macrophage and B Cell Identities. Molecular Cell 38, 576-589. doi:10.1016/j.molcel.2010.05.004

Heng, T.S.P., Painter, M.W., Immunological Genome Project Consortium, 2008. The Immunological Genome Project: networks of gene expression in immune cells. Nat. Immunol. 9, 1091-1094. doi:10.1038/ni1008-1091

Honda, K., Taniguchi, T., 2006. IRFs: master regulators of signalling by Toll-like receptors and cytosolic pattern-recognition receptors. Nat. Rev. Immunol. 6, 644-658. doi:10.1038/nri1900

Jin, Q., Yu, L.-R., Wang, L., Zhang, Z., Kasper, L.H., Lee, J.-E., Wang, C., Brindle, P.K., Dent, S.Y.R., Ge, K., 2011. Distinct roles of GCN5/PCAF-mediated H3K9ac and CBP/p300-mediated H3K18/27ac in nuclear receptor transactivation. EMBO J. 30, 249-262. doi:10.1038/emboj.2010.318

Kaikkonen, M.U., Spann, N.J., Heinz, S., Romanoski, C.E., Allison, K.A., Stender, J.D., Chun, H.B., Tough, D.F., Prinjha, R.K., Benner, C., Glass, C.K., 2013. Remodeling of the Enhancer Landscape during Macrophage Activationls Coupled to Enhancer Transcription. Molecular Cell 51, 310-325. doi:10.1016/j.molcel.2013.07.010

Kim, S., Becker, J., Bechheim, M., Kaiser, V., Noursadeghi, M., Fricker, N., Beier, E., Klaschik, S., Boor, P., Hess, T., Hofmann, A., Holdenrieder, S., Wendland, J.R., Fröhlich, H., Hartmann, G., Nöthen, M.M., Müller-Myhsok, B., Pütz, B., Hornung, V., Schumacher, J., 2014. Characterizing the genetic basis of innate immune response in TLR4-activated human monocytes. Nature Communications 5, 5236. doi:10.1038/ncomms6236

Krausgruber, T., Blazek, K., Smallie, T., Alzabin, S., Lockstone, H., Sahgal, N., Hussell, T., Feldmann, M., Udalova, I.A., 2011. IRF5 promotes inflammatory macrophage polarization and T. Nat. Immunol. 12, 231-238. doi:10.1038/ni.1990

Krausgruber, T., Saliba, D., Ryzhakov, G., Lanfrancotti, A., Blazek, K., Udalova, I.A., 2010. IRF5 is required for late-phase TNF secretion by human dendritic cells. Blood 115, 4421-4430. doi:10.1182/blood-2010-01-263020

Lavin, Y., Winter, D., Blecher-Gonen, R., David, E., Keren-Shaul, H., Merad, M., Jung, S., Amit, I., 2014. Tissue-Resident Macrophage Enhancer Landscapes Are Shaped by the Local Microenvironment. Cell 159, 1312-1326. doi:10.1016/j.cell.2014.11.018

Lee, M.N., Ye, C., Villani, A.-C., Raj, T., Li, W., Eisenhaure, T.M., Imboywa, S.H., Chipendo, P.I., Ran, F.A., Slowikowski, K., Ward, L.D., Raddassi, K., McCabe, C., Lee, M.H., Frohlich, I.Y., Hafler, D.A., Kellis, M., Raychaudhuri, S., Zhang, F., Stranger, B.E., Benoist, C.O., De Jager, P.L., Regev, A., Hacohen, N., 2014. Common genetic variants modulate pathogen-sensing responses in human dendritic cells. Science 343, 1246980-1246980. doi:10.1126/science.1246980

Lim, C.-A., Yao, F., Wong, J.J.-Y., George, J., Xu, H., Chiu, K.P., Sung, W.-K., Lipovich, L., Vega, V.B., Chen, J., Shahab, A., Zhao, X.D., Hibberd, M., Wei, C.-L., Lim, B., Ng, H.-H., Ruan, Y., Chin, K.-C., 2007. Genome-wide mapping of RELA(p65) binding identifies E2F1 as a transcriptional activator recruited by NF-kappaB upon TLR4 activation. Molecular Cell 27, 622-635. doi:10.1016/j.molcel.2007.06.038

Lone, I.N., Shukla, M.S., Charles Richard, J.L., Peshev, Z.Y., Dimitrov, S., Angelov, D., 2013. Binding of NF- $\kappa$ B to Nucleosomes: Effect of Translational Positioning, Nucleosome Remodeling and Linker Histone H1. PLoS Genet. 9, e1003830. doi:10.1371/journal.pgen.1003830

Matsuyama, T., Kimura, T., Kitagawa, M., Pfeffer, K., Kawakami, T., Watanabe, N., Kündig, T.M., Amakawa, R., Kishihara, K., Wakeham, A., 1993. Targeted disruption of IRF-1 or IRF-2 results in 
abnormal type I IFN gene induction and aberrant lymphocyte development. Cell 75, 83-97.

McAndrew, M.J., Gjidoda, A., Tagore, M., Miksanek, T., Floer, M., 2016. Chromatin Remodeler Recruitment during Macrophage Differentiation Facilitates Transcription Factor Binding to Enhancers in Mature Cells. J. Biol. Chem. 291, 18058-18071. doi:10.1074/jbc.M116.734186

Miyamoto, M., Fujita, T., Kimura, Y., Maruyama, M., Harada, H., Sudo, Y., Miyata, T., Taniguchi, T., 1988. Regulated expression of a gene encoding a nuclear factor, IRF-1, that specifically binds to IFN-beta gene regulatory elements. Cell 54, 903-913.

Murray, P.J., Allen, J.E., Biswas, S.K., Fisher, E.A., Gilroy, D.W., Goerdt, S., Gordon, S., Hamilton, J.A., Ivashkiv, L.B., Lawrence, T., Locati, M., Mantovani, A., Martinez, F.O., Mege, J.-L., Mosser, D.M., Natoli, G., Saeij, J.P., Schultze, J.L., Shirey, K.A., Sica, A., Suttles, J., Udalova, I., van Ginderachter, J.A., Vogel, S.N., Wynn, T.A., 2014. Macrophage Activation and Polarization: Nomenclature and Experimental Guidelines. Immunity 41, 14-20. doi:10.1016/j.immuni.2014.06.008

Negishi, H., Ohba, Y., Yanai, H., Takaoka, A., Honma, K., Yui, K., Matsuyama, T., Taniguchi, T., Honda, K., 2005. Negative regulation of Toll-like-receptor signaling by IRF-4. Proc. Natl. Acad. Sci. U.S.A. 102, 15989-15994. doi:10.1073/pnas.0508327102

Nutt, S.L., Metcalf, D., D'Amico, A., Polli, M., Wu, L., 2005. Dynamic regulation of PU.1 expression in multipotent hematopoietic progenitors. J. Exp. Med. 201, 221-231. doi:10.1084/jem.20041535

Olson, M.C., Scott, E.W., Hack, A.A., Su, G.H., Tenen, D.G., Singh, H., Simon, M.C., 1995. PU. 1 is not essential for early myeloid gene expression but is required for terminal myeloid differentiation. Immunity 3, 703-714.

Ostuni, R., Piccolo, V., Barozzi, I., Polletti, S., Termanini, A., Bonifacio, S., Curina, A., Prosperini, E., Ghisletti, S., Natoli, G., 2013. Latent Enhancers Activatedby Stimulation in Differentiated Cells. Cell 152, 157-171. doi:10.1016/j.cell.2012.12.018

Ouyang, X., Negishi, H., Takeda, R., Fujita, Y., Taniguchi, T., Honda, K., 2007. Cooperation between MyD88 and TRIF pathways in TLR synergy via IRF5 activation. Biochem. Biophys. Res. Commun. 354, 1045-1051. doi:10.1016/j.bbrc.2007.01.090

Ramirez-Carrozzi, V.R., Braas, D., Bhatt, D.M., Cheng, C.S., Hong, C., Doty, K.R., Black, J.C., Hoffmann, A., Carey, M., Smale, S.T., 2009. A unifying model for the selective regulation of inducible transcription by CpG islands and nucleosome remodeling. Cell 138, 114-128. doi:10.1016/j.cell.2009.04.020

Ramirez-Carrozzi, V.R., Nazarian, A.A., Li, C.C., Gore, S.L., Sridharan, R., Imbalzano, A.N., Smale, S.T., 2006. Selective and antagonistic functions of SWI/SNF and Mi-2beta nucleosome remodeling complexes during an inflammatory response. Genes Dev. 20, 282-296. doi:10.1101/gad.1383206

Ryzhakov, G., Eames, H.L., Udalova, I.A., 2015. Activation and Function of Interferon Regulatory Factor 5. Journal of Interferon \& Cytokine Research 35, 71-78. doi:10.1089/jir.2014.0023

Saccani, S., Pantano, S., Natoli, G., 2001. Two waves of nuclear factor kappaB recruitment to target promoters. J. Exp. Med. 193, 1351-1359.

Saliba, D.G., Heger, A., Eames, H.L., Oikonomopoulos, S., Teixeira, A., Blazek, K., Androulidaki, A., Wong, D., Goh, F.G., Weiss, M., Byrne, A., Pasparakis, M., Ragoussis, J., Udalova, I.A., 2014. IRF5:RelA Interaction Targets Inflammatory Genes in Macrophages. CellReports 8, 1308-1317. doi:10.1016/j.celrep.2014.07.034

Satoh, T., Takeuchi, O., Vandenbon, A., Yasuda, K., Tanaka, Y., Kumagai, Y., Miyake, T., Matsushita, K., Okazaki, T., Saitoh, T., Honma, K., Matsuyama, T., Yui, K., Tsujimura, T., Standley, D.M., Nakanishi, K., Nakai, K., Akira, S., 2010. The Jmjd3-Irf4 axis regulates M2 macrophage polarization and host responses against helminth infection. Nat. Immunol. 11, 936-944. doi:10.1038/ni.1920

Schiltz, R.L., Mizzen, C.A., Vassilev, A., Cook, R.G., Allis, C.D., Nakatani, Y., 1999. Overlapping but distinct patterns of histone acetylation by the human coactivators $\mathrm{p} 300$ and PCAF within nucleosomal substrates. J. Biol. Chem. 274, 1189-1192.

Schmidt, S.V., Krebs, W., Ulas, T., Xue, J., Baßler, K., Günther, P., Hardt, A.-L., Schultze, H., Sander, J., Klee, K., Theis, H., Kraut, M., Beyer, M., Schultze, J.L., 2016. The transcriptional regulator network of human inflammatory macrophages is defined by open chromatin. Nature Publishing Group 26, 151-170. doi:10.1038/cr.2016.1

Schultz, D.C., Ayyanathan, K., Negorev, D., Maul, G.G., Rauscher, F.J., 2002. SETDB1: a novel KAP-1associated histone $\mathrm{H} 3$, lysine 9-specific methyltransferase that contributes to HP1-mediated silencing of euchromatic genes by KRAB zinc-finger proteins. Genes Dev. 16, 919-932. doi:10.1101/gad.973302 
Seneviratne, A.N., Edsfeldt, A.O., Cole, J.E., Kassiteridi, C., Swart, M., Park, I., Green, P., Khoyratty, T.E., Saliba, D.G., Goddard, M.E., Sansom, S.N., Goncalves, I., Krams, R., Udalova, I.A., Monaco, C., 2017. Interferon Regulatory Factor 5 Controls Necrotic Core Formation in Atherosclerotic Lesions by Impairing Efferocytosis. Circulation CIRCULATIONAHA.117.027844. doi:10.1161/CIRCULATIONAHA.117.027844

Soufi, A., Garcia, M.F., Jaroszewicz, A., Osman, N., Pellegrini, M., Zaret, K.S., 2015. Pioneer Transcription Factors Target Partial DNA Motifs on Nucleosomes to Initiate Reprogramming. Cell 161, 555-568. doi:10.1016/j.cell.2015.03.017

Sripathy, S.P., Stevens, J., Schultz, D.C., 2006. The KAP1 corepressor functions to coordinate the assembly of de novo HP1-demarcated microenvironments of heterochromatin required for KRAB zinc finger protein-mediated transcriptional repression. Mol. Cell. Biol. 26, 8623-8638. doi:10.1128/MCB.00487-06

Takaoka, A., Yanai, H., Kondo, S., Duncan, G., Negishi, H., Mizutani, T., Kano, S.-I., Honda, K., Ohba, Y., Mak, T.W., Taniguchi, T., 2005. Integral role of IRF-5 in the gene induction programme activated by Toll-like receptors. Nature 434, 243-249. doi:10.1038/nature03308

Tanaka, N., Kawakami, T., Taniguchi, T., 1993. Recognition DNA sequences of interferon regulatory factor 1 (IRF-1) and IRF-2, regulators of cell growth and the interferon system. Mol. Cell. Biol. 13, 4531-4538.

van Arensbergen, J., van Steensel, B., Bussemaker, H.J., 2014. In search of the determinants of enhancer-promoter interaction specificity. Trends Cell Biol. 24, 695-702. doi:10.1016/j.tcb.2014.07.004

Wathelet, M.G., Lin, C.H., Parekh, B.S., Ronco, L.V., Howley, P.M., Maniatis, T., 1998. Virus infection induces the assembly of coordinately activated transcription factors on the IFN-beta enhancer in vivo. Molecular Cell 1, 507-518.

Weiss, M., Blazek, K., Byrne, A.J., Perocheau, D.P., Udalova, I.A., 2013. IRF5 is a specific marker of inflammatory macrophages in vivo. Mediators Inflamm. 2013, 245804-9. doi:10.1155/2013/245804

Weiss, M., Byrne, A.J., Blazek, K., Saliba, D.G., Pease, J.E., Perocheau, D., Feldmann, M., Udalova, I.A., 2015. IRF5 controls both acute and chronic inflammation. Proc. Natl. Acad. Sci. U.S.A. 112, 11001-11006. doi:10.1073/pnas.1506254112

Yanai, H., Chen, H.-M., Inuzuka, T., Kondo, S., Mak, T.W., Takaoka, A., Honda, K., Taniguchi, T., 2007. Role of IFN regulatory factor 5 transcription factor in antiviral immunity and tumor suppression. Proc Natl Acad Sci USA 104, 3402-3407. doi:10.1073/pnas.0611559104

Yie, J., Merika, M., Munshi, N., Chen, G., Thanos, D., 1999. The role of HMG I(Y) in the assembly and function of the IFN-beta enhanceosome. EMBO J. 18, 3074-3089. doi:10.1093/emboj/18.11.3074

Zhu, Y., Sun, L., Chen, Z., Whitaker, J.W., Wang, T., Wang, W., 2013. Predicting enhancer transcription and activity from chromatin modifications. Nucleic Acids Res. 41, 10032-10043. doi:10.1093/nar/gkt826 
Inflammatory $M \varphi$

Homeostatic $M \varphi$

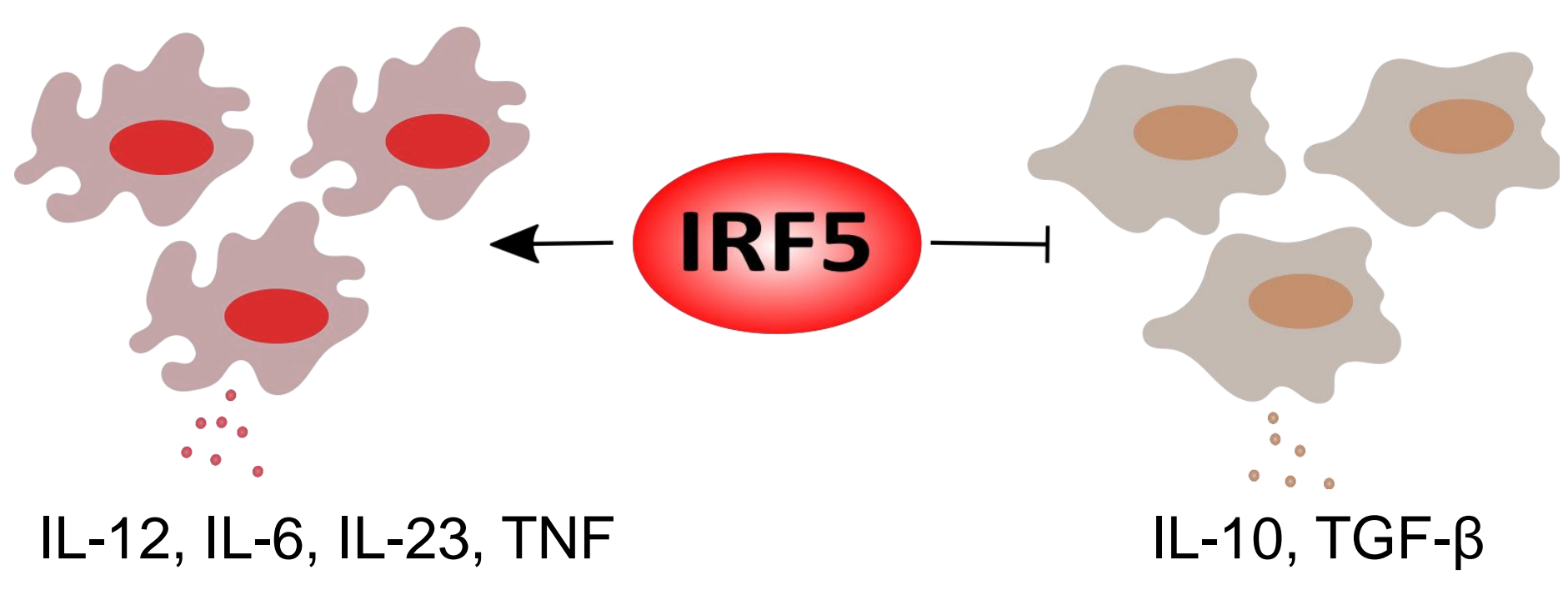

Figure 1. IRF5 is a key mediator of inflammatory macrophages. IRF5 induction leads to TNF, IL-6, IL-12 and IL-23 secretion whilst simultaneously repressing IL-10, TGF- $\beta$. In vitro macrophages can be polarised to a classic (M1) state or alternatively activated (M2) phenotype by culture with either LPS and IFN- $\gamma$ or IL-4 and IL-13. IFN- $\gamma$ and GM-CSF induce expression of IRF5 mRNA, while LPS induces post-translational modifications of IRF5 protein. 
